\title{
Brief Account of Multilayer Portal Model for Information Grid $_{1}$ \\ Mingji Chen, Xiaowu Chen, Xixi Luo \\ VRLab, School of Computer Science and Engineering, Beihang University \\ Beijing 100083, P.R. China. chen@buaa.edu.cn
}

\begin{abstract}
University Digital Museum Grid (UDMGrid) is a typical system of information grid, whose portal is to seamlessly access digital specimens by a one-stop information service. Based on information grid, semantic web and portal model, this paper presents a multilayer portal model for information grid. It is composed of six layers, including grid resource layer, grid service layer, portlet service layer, portlet layer and user layer. The application of this multilayer portal model in UDMGrid demonstrates its usability, maintainability and expansibility.
\end{abstract}

\section{Categories and Subject Descriptors}

D.2.11 [Software Engineering]: Software Architectures Domain-specific architectures, patterns, information hiding.

\section{General Terms}

Measurement, Design.

\section{Keywords}

Information grid, portal model, ontology, portlet

\section{INTRODUCTION}

UDMGrid (University Digital Museum Grid) is an information grid, which is intent to integrate the enormous dispersed resources of thirteen digital museums and to provide appropriate information services to users according to their knowledge levels and motivations via a unified grid portal. So Portal is a very key component of UDMGrid.

Grid portal is the point of access to a grid system. In order to hide the complexity of grid environment, grid portal is built upon the generic web portal model, providing a way for grid users to access these services transparently via a friendly portal interface. The well-known grid portal projects contain Grid Portal Development Kit (GPDK), Gridsphere, etc.

For information grid that has enormous information resources like UDMGrid, how to resolve the semantic heterogeneity among information resources is a great challenge. Ontology which is a key component of semantic web technology provides a way of establishing the relationships between information resources and concepts of ontology to resolve the semantic heterogeneity among these information resources, helping people to retrieve, extract, explain and process information. Semantic portal is a typical application of semantic web technology, which utilizes the semantic web technology to provide semantic search, browsing, and integration of content. The well-known semantic portal projects contain OntoWeb Portal, Museum Finland Portal, etc.

Based on information grid, semantic web and portal model, this paper presents a multilayer portal model for information grid. The application of UDMGrid demonstrates its usability, maintainability and expansibility.

\section{A MULTILAYER PORTAL MODEL}

This paper presents a multilayer portal model for information grid as shown in Figure 1.

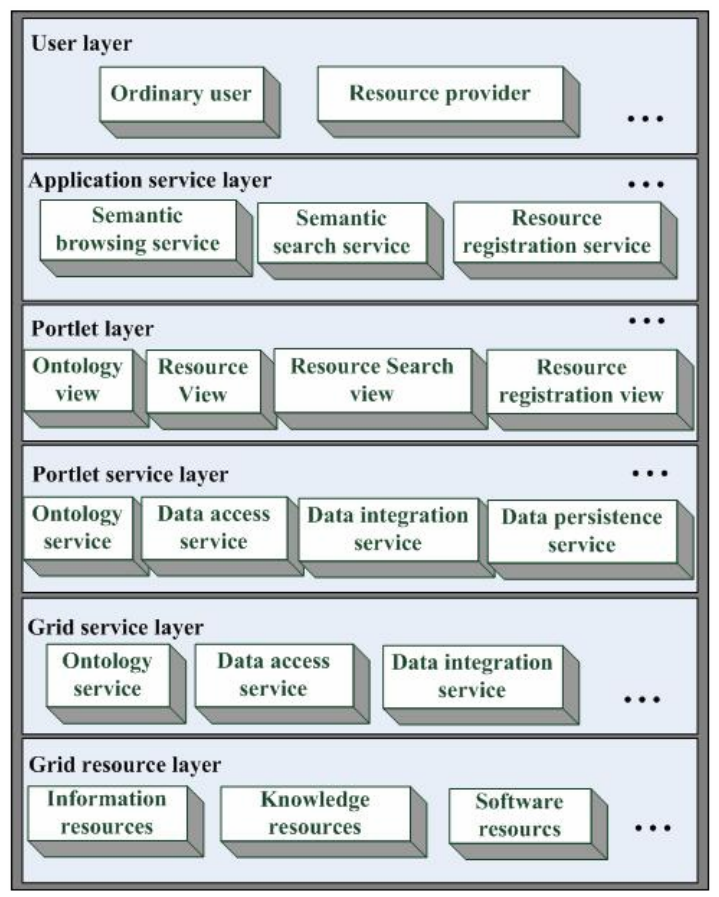

Figure 1. The multilayer portal model

Each layer is as the producer of the last layer and the consumer of the next layer as well, except for the top layer and the bottom layer. Meanwhile, each layer can only interact with adjacent layers according to the APIs. The delamination method of this portal model makes information grid portals constructed by this model have good maintainability and expansibility.

Grid resource layer consists of the distributed and heterogeneous resources that will be integrated by information grid, including information resources, knowledge resources, software resources etc. Information resources are various information sets, which can

1 This paper is supported by China Education and Research Grid Program (ChinaGrid), National Natural Science Foundation of China (60503066), National Grand Fundamental Research 973 Program of China (2006CB303000), National High-Tech Research and Development Plan of China (2006AA01Z311), National Research Foundation (5140*305*), Program for New Century Excellent Talents in University (NCET-05-0187), Program for Beijing New Stars in Science \& Technology (200411A).

Permission to make digital or hard copies of all or part of this work for personal or classroom use is granted without fee provided that copies are not made or distributed for profit or commercial advantage and that copies bear this notice and the full citation on the first page. To copy otherwise, or republish, to post on servers or to redistribute to lists, requires prior specific permission and/or a fee.

INFOSCALE 2007, June 6-8, Suzhou, China

Copyright $@ 2007$ ICST 978-1-59593-757-5

DOI 10.4108/infoscale.2007.961 
be stored in the form of databases, web pages, file systems, etc. Knowledge resources include ontology repository typically. Ontology repository stores the definition of concepts, the relationships among concepts and the relationships among concepts and their instances. It is the foundation of information integration and retrieval based on semantics. Software resources can include a series of software tools which can assist the construction of the layers upwards.

Grid service layer wraps resources in grid resource layer into grid services and provides access for portlet services. As the information grid which has enormous information resources, it typically includes ontology service, data access service, data integration service, etc. Ontology service provides the operation capabilities to ontology repository, and data access service provides the data access capabilities, including heterogeneous database access, web page access, file access, etc. Data integration service is used to integrate the resources, which are got by data access service, into a uniform resource description according to the specification of ontology.

Portlet services layer wraps grid services into portlet service so as to provide a consistent access interface to grid services which may be based on different grid middleware, and portlet services can also encapsulate application logics that can be reused among portlet. It simplifies the application logics in portlet and enhances the security and maintainability of the system.

Portlet layer includes ontology view, resource view, resource search view, resource registration view, etc. Ontology view presents all the concepts and relationships among concepts, not only can provide a global view of the resources categorized by ontology, but also a navigation guide for users to browse the resources. Resource view shows the resources in information grid, which may come from databases or web pages. Resource search view provides a mode to construct search model according to ontology. And resource registration view provides users the capability of registering the information resources into information grid according to specification of ontology.

Application service layer provides application services that satisfy users' needs. Application service can be composed of one or more portlets which can either be correlative or independent. Application services include semantic browsing service, semantic search service, resources registration service, etc. Semantic browsing service is a direct way of resource browsing, which is brought forward by the reason that people have difficulty in expressing their requirement exactly by the combination of key words. Therefore, users do not need to input their query conditions directly, but only to reduce the query bound gradually according to the organization structure of the resources and finding out the resources they need at last. Semantic search service enhances the existing search engines by adding some semantic related information such as certain background information or semantic relationships to simple keywords, making the information retrieval not just based on simple keywords matching, therefore filtering unrelated information from result set. Resource registration service provides the capability to register information resources into information grid according to the specification of ontology.
User layer is the end users of the application services provided by application service layer. According to partition rule that whether the user can provide resources, it can be divided into two kinds. One is ordinary users who only use application services but not provide resources; the other is resource providers who not only use application services but also provide resources.

\section{APPLICATION}

Adopting the multilayer portal model for information grid, we have designed and implemented the portal of UDMGrid, as shown in Figure 2, Figure 3.

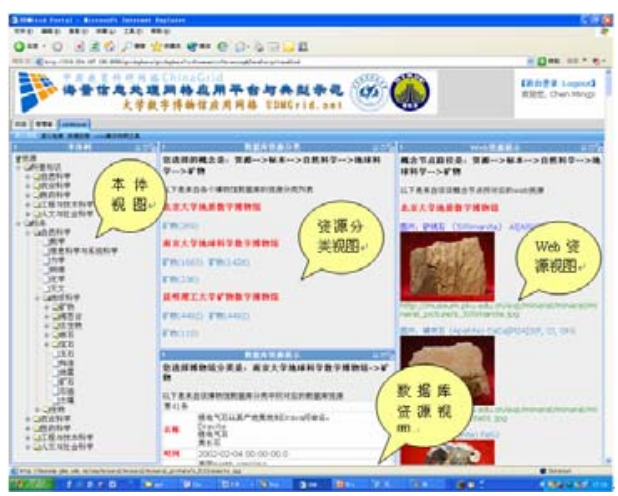

Figure 2. Semantic browsing service

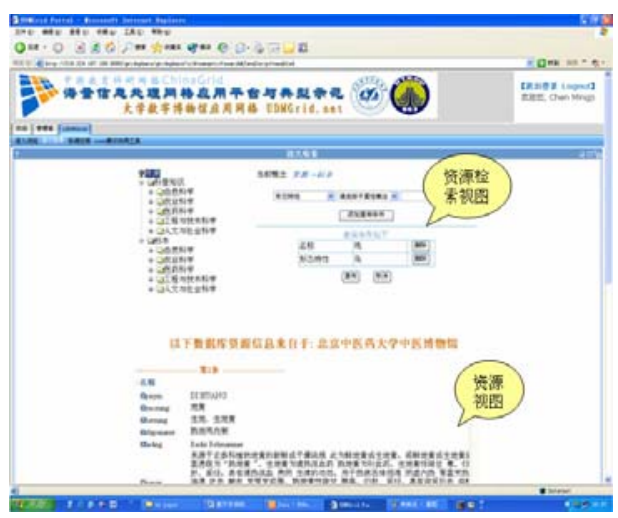

Figure 3. Semantic search service

In order to add a new museum resources to UDMGrid, the museum owners should use resource registration service to annotate their resources, the grid service developers need to modify the data access service to add new database, and the portal developers need to modify the portlet service corresponding to data access service in grid service layer, and there is no need to modify any other modules. The application service in UDMGrid such as semantic browsing service and semantic search service has considered different users' knowledge levels and motivation, providing good usability. 